\title{
Saturated duration for dynamic structural plastic response and fundamental periods of elastic vibration
}

\author{
Y.-P. Zhao
}

\begin{abstract}
The relationship is determined between saturated duration of rectangular pressure pulses applied to rigid, perfectly plastic structures and their fundamental periods of elastic vibration. It is shown that the ratio between the saturated duration and the fundamental period of elastic vibration of a structure is dependent upon two factors: the first one is the slenderness or thinness ratio of the structure; and the second one is the square root of ratio between the Young's elastic modulus and the yield stress of the structural material. Dimensional analysis shows that the aforementioned ratio is one of the basic similarity parameters for elastic-plastic modeling under dynamic loading.
\end{abstract}

Einschwingverhalten infolge dynamischer, strukturbedingt plastischer Vorgänge und den zugehörigen Grundfrequenzen der elastischen Schwingungen

Zusammenfassung In diesem Artikel wird das

Einschwingverhalten beschrieben, das sich aus dem

Einwirken von Druckstößen auf starre, ideal plastische Strukturen und den zugehörigen elastischen Schwingungen ergibt. Es wird aufgezeigt, daß das Verhältnis zwischen der Einschwingdauer und der Grundperiode elastischer Schwingungen der betrachteten Struktur von zwei Einflußgrößen bestimmt wird. Die eine ist der Schlankheitsgrad der Struktur und die andere die Quadratwurzel des Verhältnisses aus dem Elastizitätsmodul und der Fließspannung des Materials. Mit Hilfe einer Dimensionsanalyse wird ermittelt, daß der eingeführte Verhältniswert eine der grundlegenden Ähnlichkeitsgrößen für die Beschreibung des elastisch-plastischen Verhaltens bei dynamischer Belastung darstellt.

$\begin{array}{ll}\text { List of symbols } \\ B & \begin{array}{l}\text { half width of rectangular plate, radius of circular } \\ \text { plate or half span of beam }\end{array} \\ D & E H^{3} / 12\left(1-v^{2}\right), \text { flexural rigidity of thin plate } \\ \text { Dn } & \text { Johnson's damage number } \\ E & \begin{array}{l}\text { Young's elastic modulus } \\ E I\end{array} \\ \text { flexural rigidity of beam, } I=H^{3} / 12 \text { for uniform } \\ \text { beam with unit width } \\ \text { thickness of beam or plate }\end{array}$

Received: 3 May 1998

\section{Y.-P. Zhao}

Laboratory for Nonlinear Mechanics of Continuous Media (LNM), Institute of Mechanics, Chinese Academy of Sciences, Beijing 100080, People's Republic of China e-mail:yzhao@Inm.imech.ac.cn half length of rectangular plate

$\rho H$, mass per unit length of beam

magnitude of applied rectangular pressure pulse fundamental period of elastic vibration of a structure permanent central displacement of a structure $B / L$, aspect ratio of rectangular plate

Poisson's ratio

$\beta \tan \phi$ mass density uniaxial yield stress duration of rectangular pressure pulse saturated duration of rectangular pressure pulse $\tan ^{-1}\left(-\beta+\sqrt{3+\beta^{2}}\right)$

fundamental frequency of free elastic vibration

$\omega_{11}$ subscripts

$f$ final

$m \quad$ model

sat saturated

p prototype

1

\section{Introduction}

Rectangular pressure pulse (shown in Fig. 1) and impulsive loading are two most widely used idealized dynamic loads in the analyses of dynamic plasticity, and the latter one can be considered the limit case of the former one when the ratio between the magnitude of the dynamic pulse and the collapse load of the structure is sufficiently large and the pulse duration is sufficiently short, thus the study of the dynamic behavior of solids subjected to rectangular pressure pulses is of great importance.

The saturated impulses were first explicity determined by Zhao et al. [1] for the dynamic plastic response of both simply supported and fully clamped rigid-perfectly plastic beams, which are subjected to rectangular pressure pulse in the medium range, the approximate square yield conditions were adopted and the secondary effect of finitedeflections was taken into account. Later, the concept of saturated impulse was entended by Zhao et al. [2] to analyze the dynamic plastic response of other structures such as simply supported circular plate, simply supported and fully clamped square plates, and cylindrical shells subjected to the same kind of loading. As a matter of fact, saturated impulse for rectangular pressure pulse is equivalent to saturated duration of the pulse when the magnitude is given, so the corresponding dimensionless saturated duration was also given for these kinds of 


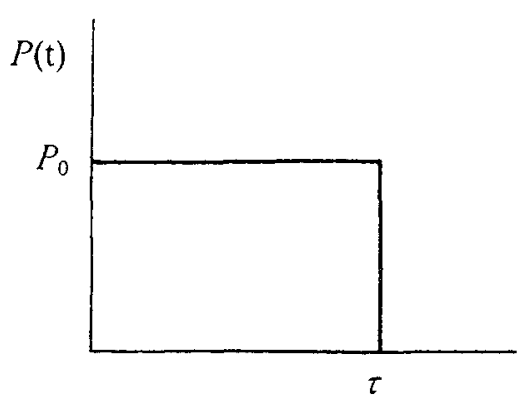

Fig. 1. Rectangular pressure pulse

structures [2]. Zhu and Yu [3] extended this concept to pulse-loaded elastic-plastic square plates. Zhao [4] has recently obtained the saturated duration of rectangular pressure pulses applied to both simply supported and fully clamped rectangular plates (shown in Figs. 2 and 3) with finite-deflections. It should be noted that the rigid-perfectly plastic results for simply supported beam, simply supported square plate and simply supported circular plate are all special cases of those of simply supported rectangular plate subjected to the same rectangular pressure pulses, and the rigid-perfectly plastic results for both fully clamped beam and fully clamped square plate are special cases of those of fully clamped rectangular plate subjected to the same rectangular pressure pulses. Thus the determination of the saturated duration of rectangular pressure pulse for rectangular plate has a more general meaning.

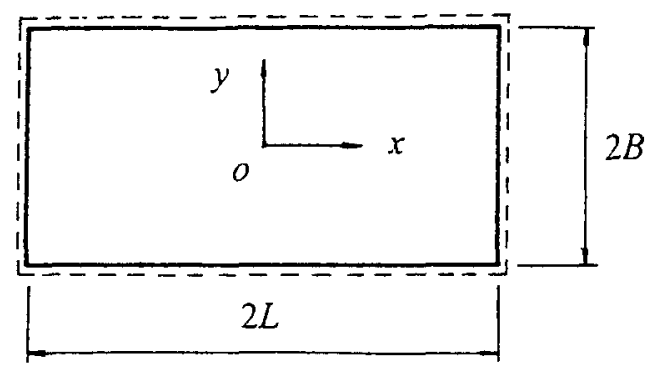

Fig. 2. Simply supported rectangular plate

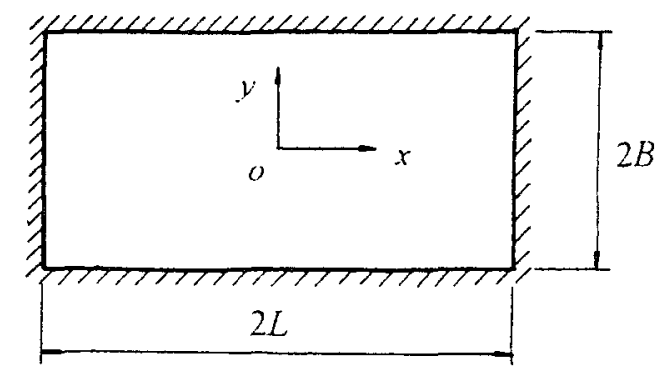

Fig. 3. Fully clamped rectangular plate
Rigid, perfectly plastic analysis is, of course, an indispensable tool in the many situations where it is appropriate. In dynamic problems it is best suited to very short pulses [5]. By using a simple mass-spring model, reference [5] studied the upper bound of the error of rigid, perfectly plastic solution relative to elastic-plastic one for many kinds of dynamic loads (including rectangular pressure pulse, half-sine force pulse, linearly decreasing pulse, triang pulse and so on), it has been found that this error for such dynamic pulses can be estimated by the combination of two factors: the first one is the energy ratio $R$, defined as plastic work divided by elastic energy capacity; the second one is the ratio between the pulse duration and the fundamental period of elastic vibration of the structure. To assist the understanding of error analysis of the rigid, perfectly plastic model, the major aim of the present paper is to obtain the ratio between the saturated duration of rectangular pressure pulse $\tau_{\text {sat }}$ and the fundamental period of elastic vibration $T$ for some kinds of structures such as simply supported or fully clamped beams, rectangular and square plates which are simply supported or fully clamped around their boundaries, and simply supported circular plate.

\section{2 \\ Saturated duration and fundamental period of elastic vibration}

The saturated duration of rectangular pressure pulses applied to both simply supported and fully clamped beams is [2]

$\tau_{\mathrm{sat}}=\pi B \sqrt{\frac{\rho}{3 \sigma_{0}}}$,

where $\rho$ and $\sigma_{0}$ are mass density and uniaxial yield stress of the beam material, respectively, $B$ is half span of the beams. Equation (1) means, that the saturated duration of the rectangular pressure pulse is proportional to the length of the beam, and it is also proportional to the square root of the ratio between the mass density and the yield stress of the beam material. Equation (1) also implies, that the saturated duration depends neither on the magnitude of the dynamic pulse, nor on the thickness of the beams.

The saturated duration of the rectangular pressure pulses applied to simply supported and fully clamped square plates as well as simply supported circular plate is given by [2]

$\tau_{\text {sat }}=\pi B \sqrt{\frac{\rho}{6 \sigma_{0}}}$,

where $B$ is the semi-width of the square plate or the radius of the circular plate. Both (1) and (2) have the same characteristics.

The fundamental period of elastic vibration $(T)$ is [6]

$T=\frac{8 B^{2}}{\pi}\left(\frac{m}{E I}\right)^{1 / 2}$,

and

$T=\frac{8 \pi B^{2}}{4.73^{2}}\left(\frac{m}{E I}\right)^{1 / 2}$, 
for uniform beams, which are simply supported and fully clamped. $m$ is the mass per unit length of the beam, and $E I$ denotes the rigidity of the beam. For a beam with unit width, we have

$m=\rho H, I=\frac{H^{3}}{12}$.

The ratio of the saturated duration to the fundamental period of vibration is

$\frac{\tau_{\text {sat }}}{T}=\frac{\pi^{2}}{48} \frac{H}{B} \sqrt{\frac{E}{\sigma_{0}}} \approx 0.21 \frac{H}{B} \sqrt{\frac{E}{\sigma_{0}}}$,

and

$\frac{\tau_{\text {sat }}}{T} \approx 0.47 \frac{H}{B} \sqrt{\frac{E}{\sigma_{0}}}$,

for a simply supported or fully clamped beam, respectively. Both (6) and (7) show that the ratio $\tau_{\text {sat }} / T$ is proportional to the slenderness ratio of the beam, and it is also proportional to the square root of the ratio between the Young's elastic modulus and the yield stress of the beam material.

For aluminium alloy the uniaxial yield stress is in the range $\sigma_{0}=100 \sim 630 \mathrm{MPa}$, and the Young's elastic modulus is in the range $E=69 \sim 79 \mathrm{GPa}$, in such case we have

$\sqrt{\frac{E}{\sigma_{0}}} \approx 10 \sim 26.6$.

If half the slenderness ratio of a beam is chosen to be $H / B \approx 1 / 47$ [7], then from (6) and (7) we have the range of $\tau_{\text {sat }} / T$ as follows

$\frac{\tau_{\text {sat }}}{T} \approx 0.045 \sim 0.12$,

and

$\frac{\tau_{\text {sat }}}{T} \approx 0.01 \sim 0.27$,

for simply supported and fully clamped beams, respectively.

For mild steel, if the ratio between yield stress and Young's modulus is chosen to be $\sigma_{0} / E \approx 5.0 \times 10^{-3}$, and for the same half slenderness ratio of the beam

$H / B \approx 1 / 47$, we have $\tau_{\text {sat }} / T \approx 0.063$ and $\tau_{\text {sat }} / T \approx 0.14$ for simply supported and fully clamped beams, respectively.

The fundamental period of elastic vibration of a simply supported rectangular plate is [8]

$T=\frac{8}{\pi} \frac{L^{2} B^{2}}{L^{2}+B^{2}} \frac{1}{H} \sqrt{\frac{3\left(1-v^{2}\right) \rho}{E}}$,

where $v$ is the Poisson's ratio, $L$ and $B$ are the semi-length and semi-width of the plate, respectively. The saturated duration of the applied rectangular pressure pulse in the medium range applied to rectangular plate as follows [4]

$\tau_{\text {sat }}=\pi B \sqrt{\frac{\rho}{6 \sigma_{0}}} \sqrt{\frac{\left(3-2 \xi_{0}\right)\left(2-\xi_{0}\right)}{1+\left(1-\xi_{0}\right)\left(2-\xi_{0}\right)}}$,

where

$\xi_{0}=\beta \tan \phi, \tan \phi=-\beta+\sqrt{3+\beta^{2}}, \beta=B / L$.

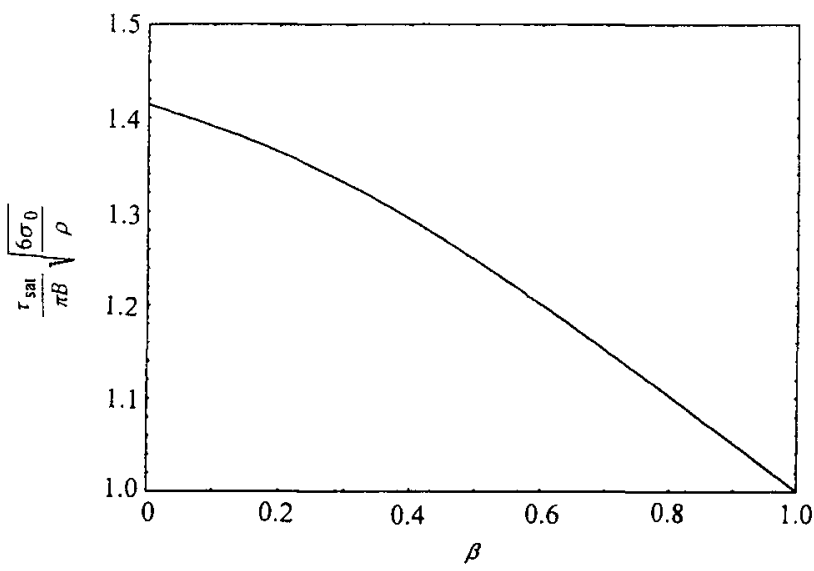

Fig. 4. Relationship between dimensionless saturated duration and aspect ratio for rectangular plate

The relationship between the dimensionless saturated duration $\frac{\tau_{s a b}}{\pi B} \sqrt{\frac{6 \sigma_{0}}{\rho}}$ and the aspect ratio $\beta$ of the rectangular plate is shown in Fig. 4. Figure 4 illustrates that the dimensionless saturated duration $\frac{\tau_{s a t}}{\pi B} \sqrt{\frac{\sigma_{0}}{\rho}}$ decreases monotonically with the increase of the aspect ratio $\beta$ of the rectangular plate. For simply supported square plate $\beta=1$ (one limit point in Fig. 4 ), the saturated duration of the rectangular pressure pulse in the medium range is then reduced to (2). It is well-known that the solution of a simply supported, rigid-perfectly plastic circular plate is the same as that of a simply supported, rigid-perfectly plastic square plate subjected to the same rectangular pressure pulses, then (2) is also the saturated duration of rectangular pressure pulse for the dynamic plastic response of a simply supported circular plate with radius $B$. It is also interesting that simply supported beam is a special case when $\beta=0$ (another limit point in Fig. 4), then from (13) and (12) we can get the saturated duration of rectangular pressure pulse for the dynamic plastic response of a simply supported, rigid-perfectly plastic beam as (1).

The ratio $\tau_{\text {sat }} / T$ for a simply supported rectangular plate is

$$
\frac{\tau_{\text {sat }}}{T}=\frac{\pi^{2}}{24}\left(1+\beta^{2}\right) \frac{H}{B} \sqrt{\frac{E}{2\left(1-v^{2}\right) \sigma_{0}}} \sqrt{\frac{\left(3-2 \xi_{0}\right)\left(2-\xi_{0}\right)}{1+\left(1-\xi_{0}\right)\left(2-\xi_{0}\right)}} .
$$

For square plate $L=B,(13)$ gives $\beta=1$ and $\xi_{0}=1$, then from (14) we have the ratio between the saturated duration of a simply supported square and the fundamental period of elastic vibration $\left(\tau_{\text {sat }} / T\right)$ is

$$
\frac{\tau_{\text {sat }}}{T}=\frac{\pi^{2}}{12} \frac{H}{B} \sqrt{\frac{E}{2\left(1-v^{2}\right) \sigma_{0}}} .
$$

For common cases we can set $v=0.3$, then (15) is reduced to

$$
\frac{\tau_{\mathrm{sat}}}{T}=0.61 \frac{H}{B} \sqrt{\frac{E}{\sigma_{0}}} .
$$


Similarly, for mild steel, if the ratio between yield stress and Young's modulus is chosen to be $\sigma_{0} / E \approx 5.0 \times 10^{-3}$, and half thinness ratio of the simply supported square plate is chosen to be $H / B=0.042$ [9], then from (16) we have

$\frac{\tau_{\text {sat }}}{T} \approx 0.36$

If $H / B=1 / 40$, then we have $\tau_{\text {sat }} / T \approx 0.22$.

For a fully clamped square plate, the fundamental period of elastic vibration is [8]

$T=\frac{8 \pi}{5.99^{2}} \frac{B^{2}}{H} \sqrt{3\left(1-v^{2}\right) \frac{\rho}{E}}$.

The ratio between the saturated duration and the fundamental period of elastic vibration of a fully clamped square plate $\left(\tau_{\text {sat }} / T\right)$ is

$\frac{\tau_{\text {sat }}}{T} \approx 1.50 \frac{H}{B} \sqrt{\frac{1}{2\left(1-v^{2}\right)} \frac{E}{\sigma_{0}}}$.

For common materials we choose $v=0.3$, then (19) is reduced to

$\frac{\tau_{\text {sat }}}{T}=1.11 \frac{H}{B} \sqrt{\frac{E}{\sigma_{0}}}$.

If $\sigma_{0} / E \approx 5.0 \times 10^{-3}$ and $H / B=0.042$ are taken for example, then we have $\tau_{\text {sat }} / T \approx 0.66$.

For a simply supported circular plate, the fundamental frequency of elastic vibration is [8]

$\omega_{11}=\frac{\lambda_{11}^{2}}{4 B^{2}} \sqrt{\frac{D}{\rho H}}$,

where $D=E H^{3} / 12\left(1-v^{2}\right)$ is the flexural rigidity of the plate. If $v=0.3$, then $\hat{\lambda}_{11}=6.97$, in this case the fundamental period of elastic vibration of the simply supported circular plate is given by

$T=\frac{8 \pi}{6.97^{2}} B^{2} \sqrt{\frac{\rho H}{D}}$,

and the ratio between the saturated duration for dynamic plastic response and the fundamental period of elastic vibration $\left(\tau_{\text {sat }} / T\right)$ is $\frac{\tau_{\text {sat }}}{T}=1.50 \frac{H}{B} \sqrt{\frac{E}{\sigma_{0}}}$.

If $\sigma_{0} / E \approx 2.2 \times 10^{-3}$ and $H / B=0.31 / 60 \approx 0.0052$ are chosen [9], then from (23) we have

$\frac{\tau_{\text {sat }}}{T} \approx 0.17$,

and if $\sigma_{0} / E \approx 2.2 \times 10^{-3}$ and $H / B=1.6 / 75 \approx 0.021$ are chosen [9], then (23) gives $\tau_{\text {sat }} / T \approx 0.67$.

The main results of this section are summarized in Table 1 .

\section{3}

\section{Discussion and concluding remarks}

This paper determines the relationship between saturated duration of rectangular pressure pulses applied to rigid, perfectly plastic structures (simply supported or fully clamped beams, simply supported circular plate, simply supported or fully clamped plates) and their fundamental periods of elastic vibration. It is shown that the ratio between the saturated duration and the fundamental period of elastic vibration of a structure is dependent upon two factors: the first one is the slenderness or thinness ratio of the structure; and the second one is the square root of ratio between the Young's elastic modulus and the yield stress of the structural material.

From (1) and (2), we know that the value $B \sqrt{\rho / \sigma_{0}}$, which has the dimension of time, is expected to become a new important characteristic parameter of the dynamic plastic response of structures under rectangular pressure pulse, and the ratio $\tau / B \sqrt{\rho / \sigma_{0}}$ is expected to be an important similarity parameter for dynamic plastic modeling, in fact, for dynamic plastic response of a structure (a beam with thickness $H$ and span $2 B$ is taken as a example) subjected to rectangular pressure pulse, we have the following functional relationship

$W_{f}=F\left(p_{0}, \tau, \rho, \sigma_{0}, H, B\right)$,

where $W_{f}$ is the final displacement at the center of the beam, other symbols are defined as before. By Buckingham's $\pi$-theorem, (25) can be reduced to

$\frac{W_{f}}{H}=f\left(\mathrm{Dn}, \frac{\tau}{B \sqrt{\rho / \sigma_{0}}}, \frac{H}{B}\right)$,

Table 1. Saturated duration and fundamental period of elastic vibration. s-s: simply supported; f-c: fully clamped; the values $T$ and $\tau_{\text {sat }} / T$ for s-s circular plate are when the Poisson's ratio $v=0.3$.

\begin{tabular}{|c|c|c|c|}
\hline type of structures & $T$ & $\tau_{\mathrm{sat}}$ & $\tau_{\text {sat }} / T$ \\
\hline s-s beam & $\frac{16}{\pi} \frac{B^{2}}{H} \sqrt{3 \frac{P}{E}}$ & $\pi B \sqrt{\frac{\rho}{3 \sigma_{0}}}$ & $0.21 \frac{H}{B} \sqrt{\frac{E}{\sigma_{0}}}$ \\
\hline $\mathrm{f}-\mathrm{c}$ beam & $\frac{16 \pi}{4.73^{2}} \frac{B^{2}}{H} \sqrt{3 \frac{P}{E}}$ & $\pi B \sqrt{\frac{p}{3 \sigma_{0}}}$ & $0.47 \frac{H}{B} \sqrt{\frac{E}{c_{0}}}$ \\
\hline s-s square plate & $\frac{4}{\pi} \frac{B^{2}}{H} \sqrt{\frac{3\left(1-p^{2}\right) \rho}{E}}$ & $\pi B \sqrt{\frac{\rho}{6 \sigma_{0}}}$ & $\frac{\pi^{2}}{12} \frac{H}{B} \sqrt{\frac{\mathrm{I}}{2\left(1-\nu^{2}\right)} \frac{E}{\sigma_{0}}}$ \\
\hline $\mathrm{f}$-c square plate & $\frac{8 \pi}{5.99^{2}} \frac{B^{2}}{H} \sqrt{\frac{3\left(1-v^{2}\right) \rho}{E}}$ & $\pi B \sqrt{\frac{p}{6 \sigma_{0}}}$ & $1.50 \frac{H}{B} \sqrt{\frac{1}{2\left(1-1^{2}\right)} \frac{E}{\sigma_{0}}}$ \\
\hline s-s circular plate & $\frac{16 \pi}{6.97^{2}} \frac{B^{2}}{H} \sqrt{\frac{3\left(1-v^{2}\right) p}{E}}$ & $\pi B \sqrt{\frac{p}{6 \sigma_{0}}}$ & $1.50 \frac{H}{B} \sqrt{\frac{E}{\sigma_{0}}}$ \\
\hline
\end{tabular}


where $\mathrm{Dn}=p_{0}^{2} \tau^{2} /\left(\rho H^{2} \sigma_{0}\right)$. The first similarity parameter in above equation $\Pi_{1}=\mathrm{Dn}$ is the Johnson's damage number for rectangular pressure pulse [10], the second similarity parameter $\Pi_{2}=\frac{\tau}{B \sqrt{\rho / \sigma_{0}}}$ is the ratio between the duration of the applied pulse and the order of the saturated duration of the applied load, $\Pi_{3}=H / B$ is the order of the slenderness or thinness ratio of the structure.

For elastic-plastic dynamic modeling, the functional relationship equivalent to (25) is expressed

$W_{f}=F\left(p_{0}, \tau, \rho, E, \sigma_{0}, H, B, v\right)$,

By using Buckingham's $\pi$-theorem, we have

$\frac{W_{f}}{H}=f\left(\frac{E}{\sigma_{0}}, \mathrm{Dn}, \frac{H}{B} \sqrt{\frac{E}{\sigma_{0}}}, \frac{H}{B}, v\right)$.

If the constitutive similarity, which means that model and prototype materials have homologous constitutive properties and homologous stress-strain curves, is met $[10,11]$, then $\left(E / \sigma_{0}\right)_{m}=\left(E / \sigma_{0}\right)_{p}$, here subscripts $m$ and $p$ refer to model and prototype, respectively. Thus the first similarity parameter $\Pi_{1}=E / \sigma_{0}$ in (28) represents the requirement of constitutive similarity, geometrical similarity between model and prototype means $(H / B)_{m}=(H / B)_{p}$, so from the requirements of both geometrical and constitutive similarities, we have $\left(\frac{H}{B} \sqrt{\frac{E}{\sigma_{0}}}\right)_{m}=\left(\frac{H}{B} \sqrt{\frac{E}{\sigma_{0}}}\right)_{p}$, this is exactly the dimensionless relationship between model and prototype $\left(\tau_{\text {sat }} / T\right)_{m}=\left(\tau_{\text {sat }} / T\right)_{p}$, which is one of the essential requirements for elastic-plastic dynamic modeling.

\section{References}

1. Zhao YP, Yu TX, Fang I (1994) Large dynamic plastic deflection of a simply supported beam subjected to rectangular pressure pulse. Arch. Appl. Mech. 64, 223-232

2. Zhao YP, Yu TX, Fang J (1995) Saturated impulses for dynamically loaded structures with finite-deflections. Struct. Engng. \& Mech. 3, 583-592

3. Zhu L, Yu TX (1997) Saturated impulse for pulsed-loaded elastic-plastic square plates. Int. J. Solids Structures 34, 17091718

4. Zhao YP (1997) Saturated duration of rectangular pressure pulse applied to rectangular plates with finite-deflections. Mech. Res. Commu. 24, 559-666.

5. Symonds PS, Frye CWG (1988) On the relation between rigidplastic and elastic-plastic predictions of response to pulse loading. Int. J. Impact Engng 7, 139-149

6. Jones N (1989) Structural Impact. Cambridge University Press

7. Humphreys JS (1965) Plastic deformation of impulsively loaded straight clamped beams. ASME J. Appl. Mech. 32, 7-10

8. Cao ZY (1989) Vibration theory of plates and shells. China Railway Press

9. Nurick GN, Martin JB (1989) Deformation of thin plates subjected to impulsive loading-A review, part II: experimental studies. Int. J. Impact Engng 8, 171-186

10. Zhao YP (1998) Prediction of structural dynamic plastic shear failure by Johnson's damage number. Forsch. Ingenieurwes. $63,349-352$

11. Baker WE, Westine PS, Dodge FT (1973) Similarity methods in engineering dynamics. Hayden Book Company, Inc 